\title{
Protective Effect of Eucalyptus Oil Against Pulmonary Destruction and Inflammation in COPD Rats
}

\author{
Lin Wang ${ }^{1}$, Jianbo Sun ${ }^{2}$, Wanzhong Li ${ }^{1}$, Yanna Lv ${ }^{1}$, Weiwei Shi ${ }^{1}$, Chunzhen Zhao ${ }^{1,}$ * \\ ${ }^{1}$ Department of Pharmacology and Applied Pharmacology Laboratory, Laboratory for Cognitive Neuroscience, Weifang Medical University, \\ Weifang, China \\ ${ }^{2}$ Department of Neurosurgery, Shouguang People's Hospital, Shouguang, China
}

Email address:

chugzw@hotmail.com (Chunzhen Zhao)

${ }^{*}$ Corresponding author

\section{To cite this article:}

Lin Wang, Jianbo Sun, Wanzhong Li, Yanna Lv, Weiwei Shi, Chunzhen Zhao. Protective Effect of Eucalyptus Oil Against Pulmonary Destruction and Inflammation in COPD Rats. Journal of Diseases and Medicinal Plants. Vol. 3, No. 1, 2017, pp. 17-22. doi: $10.11648 /$ j.jdmp.20170301.14

Received: January 23, 2017; Accepted: February 13, 2017; Published: March 1, 2017

\begin{abstract}
Eucalyptus oil (EO), an essential oil isolated from Eucalyptus leaves, was examined for its effect on LPS and Klebsiella pneumoniae - induced COPD in rats. The COPD model was induced by instilling intratracheally with LPS and Klebsiella pneumoniae (K. P). The test compound, EO (30, 100 and $300 \mathrm{mg} / \mathrm{kg})$, Prednisone Acetate $(10 \mathrm{mg} / \mathrm{kg}) \mathrm{or}$ vehicle was instilled intragastrically after three weeks exposure of LPS and K. P, lasted for 4 weeks. EO significantly reduced amounts of inflammatory cells in bronchoalveolar lavage fluid (BALF) and blood, and decreased bronchiolitis, emphysematous changes and thickness of bronchioles. It also significantly reduced the increased AB-PAS-positive goblet cells in bronchioles. Prednisone Acetate attenuated pulmonary inflammation and airway mucus hypersecretion, but no significant difference was found on emphysema. Pretreatment with EO markly reduced the production of proinflammatory cytokines TNF- $\alpha$ and IL- $\beta$ in lung homogenate, significantly decreased the elevated malondialdehyde (MDA) level and and increased superoxide dismutase (SOD) activity. These findings indicate that EO could exert an protective effect against LPS plus K. P-induced lung indury via inhibition of proinflammatory cytokines production and improvement of anti-oxidant status. Our results provide evidence that EO might have its potential to be a proper candidate drug in the treatment of COPD.
\end{abstract}

Keywords: Eucalyptus Globulus, Lipopolysaccharide, Cytokine, Chronic Obstructive Pulmonary Disease

\section{Introduction}

Chronic obstructive pulmonary disease (COPD) is characterised by chronic inflammation and irreversible airflow obstruction, mainly induced by cigarette smoking and noxious stimuli including infection [1]. Chronic and persistent inflammation results in emphysema and irreversible airway narrowing that resulted from protease, mucociliary dysfunction, oxidative stress or fibrosis around small airway. There is increasing evidence that bacterial colonization in COPD patients contributed to airway inflammation and excerbated the progression of decline lung function [2-4]. However, the mechanisms are still not well known. Xu et al. succeeded to establish a COPD rat model by repeated injecting intranasally of Klebsiella pneumoniae [5], suggesting an important role of bacterial infection in the pathogenesis of COPD.

Eucalyptus essential oil (EO), is commonly used as expectorant for upper respiratory tract infection or inflammation, as well as decongestant and various other inflammatory diseases. It's reported that EO possesses particular anti-inflammatory and anti-oxidative properties [6, 7]. In murine macrophages, Eucalyptus oil inhibited inducible nitric oxide synthase mRNA expression and NO production induced by lipopolysaccharide and IFN- $\gamma$ [8]. Concomitant therapy of Eucalyptol reduced exacerbations and improves lung function in patients with COPD [9]. These findings support at least for some of the essential oils of Eucalyptus species used in the clinical treatment. However, direct evidence is still lack to identify its pharmacological proterties in chronic obstructive pulmonary diseases. Therefore, in the 
present study, we establish a rat model of COPD through intratracheal adminstration of LPS plus Klebsiella pneumonia, and determined the effects of Eucalyptus oil on pulmonary destruction and inflammatory responses.

\section{Materials and Methods}

\subsection{Animals}

Male Sprague-Dawley rats, obtained from Shanghai experimental animal center, China, weighing 180-230 g, were kept at $23 \pm 2{ }^{\circ} \mathrm{C}$ with a $12 \mathrm{~h}$ light $/ 12 \mathrm{~h}$ dark cycle. They were allowed free access to food and water. All animals were handled in accordance with the Ethical Principles for Care and Use of Laboratory Animals as previously reported (Zhao $\mathrm{CZ}$, et al., 2014). All procedures decribed herein were reviewed by and had aproved by the local animal ethics committee.

\subsection{Drugs and Reagents}

Eucalyptus oil, which was commercially prepared, was used in all of the experiments (Batch No.060711, provided by our laboratory). Klebsiella pneumoniae (No. 1.1736) was purchased from Agricultural Culture Collection of China (ACCC), LPS (Escherichia coli O111: $\mathrm{B}_{4}$ ) was purchased from Sigma.

\subsection{Experimental Procedure}

COPD model was induced by intratracheal LPS plus K. P exposure. Rats were randomly divided into seven groups, and performed as follows: $0.1 \mathrm{~mL}$ of $\mathrm{K}$. P (density, $\geq 6 \times 10^{8}$ $\mathrm{CFU} / \mathrm{mL}$ ) was instilled intratracheally twice a week and 2 $\mathrm{mg} / \mathrm{kg}$ of LPS once every two weeks; EO group (30, 100, and $300 \mathrm{mg} / \mathrm{kg}$ ) started to be administrated intragastricly after 12 weeks exposure of K. P and LPS and lasted for 4 weeks; Prednisolone Acetate (Pred, $10 \mathrm{mg} / \mathrm{kg}$ ) was administrated as positive control group, aseptic saline was administrated intragastricly as negative control group.

\subsection{Preparation of Bronchoalveolar Lavage Fluids (BALF) and Blood for Cell Counting}

Rats were anesthetized intraperitoneally with sodium pentobarbital, and BALF was harvested $24 \mathrm{~h}$ post-last $\mathrm{K}$. P and LPS exposure. Trachea of each rat was surgically exposed and cannulated. The right lungs were lavaged with $1.5 \mathrm{~mL}$ of PBS three times, fluid recovery was routinely $\geq 90 \%$. A $0.1 \mathrm{~mL}$ aliquot was used for total leukocyte number counting. $20 \mu \mathrm{L}$ of tail vein blood was harvested and added to $0.38 \mathrm{~mL}$ of $2 \%$ acetic acid solution, and stained with Wright-Giemsa staining. The total inflammatory cell number in the blood film was counted under oil immersion lens.

\subsection{Histological Examination}

The lungs were collected and fixed with $10 \%$ neutral formalin for one week. After tissues were paraffinized, $5 \mu \mathrm{m}$ sections were cut and stained with hematoxylin-eosin (H. E.) staining for evaluation of alveolar/interstitial inflammation and emphysema.

\subsection{Morphological Assessment}

After the lung tissues were paraffin-embedded and sectioned, $5 \mu \mathrm{m}$ sections were stained with $\mathrm{H}$. E. To evaluate extent of lung destruction, focus the presence of any one: (1) pulmonary mean linear intercept (Lm); (2) mean alveolar number (MAN); (3) ratio of thickness of bronchioles to diameter. Lm, as a measure of interalveolar wall distance, was computed on each slice based on 8 random fields using a cross-line under light microscopy. The total length of the cross-line divided by the numbers of the alveolar wall intersecting the lines was defined as Lm. MAN, an indicator of alveolar density, was computed on each slice based on 8 random fields by counting the numbers of alveoli and dividing the number by the area of the field. Ratio of thickness of bronchioles to diameter was computed on each slice based on 3 5 medium bronchi through measuring external diameter and internal diameter and dividing the difference by external diameter (Wang et al., 2014).

\subsection{Determination of Goblet Cell Hyperplasia}

After fixation, $5 \mu \mathrm{m}$ sections were stained with Alcian blue-Periodic Acid Schiff (AB-PAS). Positive goblet cell number and total cell number of columnar epithelial cells were counted in the main bronchus and 2 3 medium bronchi. The ratio of positive goblet cells was calculate by dividing the positive goblet cell number and total cell number of columnar epithelial (Takeyama et al., 1999). level and and increased activity

\subsection{Determination for $T N F-\alpha, I L-1 \beta, M D A$ and $S O D$ Activity in Lung Tissues}

Lung tissues stored at $-80^{\circ} \mathrm{C}$ were homogenized in homogenization Tris-buffer on ice using Heidolph Diax 900 Homogenizer (Heidolph, Germany) and then centrifuged at $12000 \mathrm{~g}$ for $30 \mathrm{~min}$ at $4^{\circ} \mathrm{C}$. The supernatants were used to determine the level of TNF- $\alpha$, IL- $1 \beta$, SOD and MDA activity. Concentration of the protein in the supernatants was detected using coomassie brilliant blue G250 method. The concentrations of TNF- $\alpha$, IL-1 $\beta$, superoxide dismutase (SOD) and malondialdehyde (MDA) were determined using commercial ELISA kits (BD Bioscience, USA; Jiancheng Bioengineering Institute, Nanjing, China). All procedures were done according to the instructions of the manufactures (Huang et al., 2013).

\subsection{Statistical Analysis}

Data were expressed as mean \pm SD and analyzed by a one-way analysis of variance (ANOVA) followed by Dennett's post hoc test with SPSS 15.0 for Windows. For all analyses, significance was calculated with a $\mathrm{P}$ value $<0.05$ considered statistically significant. 


\section{Results}

\subsection{Effect of EO on Cell Infiltration in BALF and Blood}

To determine the effect of EO on pulmonary inflammation and pheripheral inflammation, we counted the number of leukocytes in the BALF and blood. After LPS plus K. P exposure for 3 months, the total number of leukocytes in BALF and blood was significantly increased. EO at 30 and

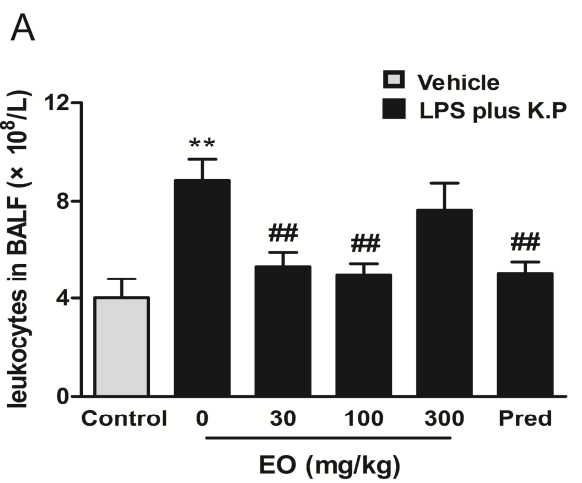

$100 \mathrm{mg} / \mathrm{kg}$ significantly decreased the infiltrated leukocytes cells in BALF and blood (Figure.1, A and B), as compared with vehicle group. Treatment with EO at $300 \mathrm{mg} / \mathrm{kg}$ also obviously decreased the number of leukocytes in BALF and blood, but no significant difference was found. Pred at 10 $\mathrm{mg} / \mathrm{kg}$ significantly reduced the cell number of leukocytes in BALF and blood.

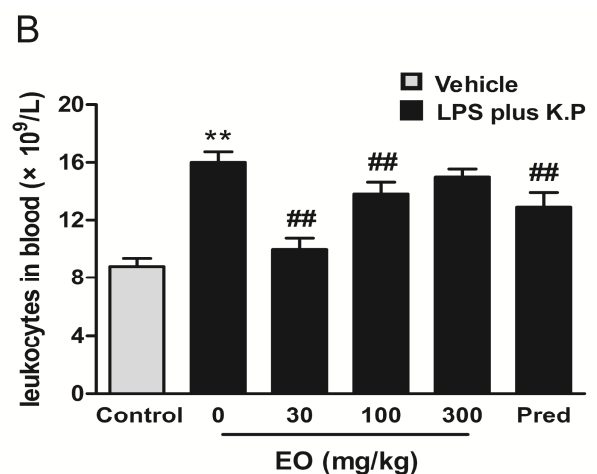

Figure 1. Effect of EO on total cell numbers of inflammatory cells in BALF and blood. 24 h post-last K. P exposure, total cell numbers in BALF (A) and blood (B) were counted and analyzed. Data were expressed as mean $\pm S D$ of $8-12$ rats /group. ${ }^{* *} P<0.01$ compared with control group, ${ }^{\#} P<0.01$ compared with group.

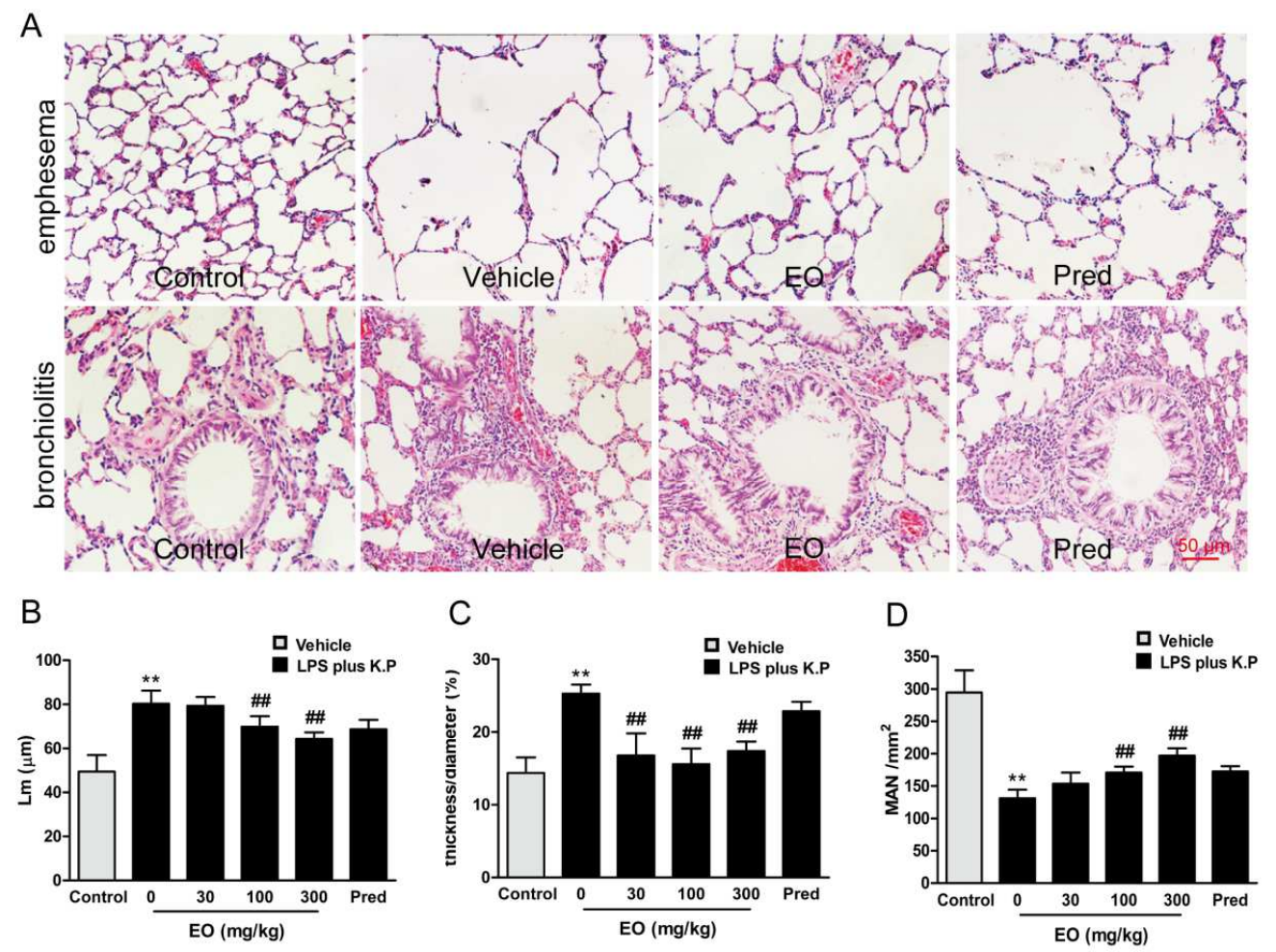

Figure 2. Effect of EO on histochemical changes in rats. Twenty-four hours after the last K. P exposure, the lungs were immersed in $10 \%$ neutral formalin for 7 days. After tissues were paraffinized, 5 m sections were prepared and stained with hematoxylin-eosin for observation of emphysema change and bronchiolitis (A). To estimate the extent of lung destruction in rats, Lm (B), thickness of bronchioles (C) and MAN (D) were evaluated as described in methods. Data were expressed as mean $\pm S D$ of 6 rats /group. ${ }^{* *} P<0.01$ compared with control group, ${ }^{\# \#} P<0.01$ compared with vehicle group.

\subsection{Effect of EO on Emphysematous Destruction and Bronchiolitis}

To observe the effect of EO on emphysema and bronchiolitis, LPS plus K. P was intratracheally instilled and induced emphysematous destruction, bronchiolitis, small airway remodeling within 4 months. In vehicle group, compared with the normal alveolar structure, the lung parenchyma was largely destructed and less alveolus was found after intratracheal instillation of LPS plus K. P; bronchiolitis was severe since a lot of inflammatory cells 
gathered around the fine bronchus. Treatment with EO and Prednisone obviously reduced emphysematous damage and bronchiolitis around the bronchioles (Figure. 2A). Histological analyses showed that $\mathrm{Lm}$ and thickness of bronchioles were significantly increased after instillation of LPS plus K. P (Figure. 2, B and C), while MAN was significantly decreased (Figure. $2 \mathrm{D}, \mathrm{P}<0.01$ ). Treatment with EO at 100 and $300 \mathrm{mg} / \mathrm{kg}$ showed less emphysematous damage, decreased thickness of bronchioles and increased MAN values compared with vehicle group. No significant difference was found between vehicle group and Pred group.

\subsection{Effect of Eucalyptus Oil on Mucus Secretion}

As shown in Fig.3A, there were more AB-PAS-positive goblet cells in bronchioles in the presence of K. P plus LPS, while less was found in Eucalyptus oil group and Prelon group. The total number of AB-PAS-positive goblet cells was significantly increased in vehicle group as compared with control group. EO significantly reduced the number of AB-PAS-positive goblet cells in bronchioles at 30, 100 and $300 \mathrm{mg} / \mathrm{kg}$ (Figure.3B). Pred $(10 \mathrm{mg} / \mathrm{kg}$ ) also significantly reduced the number of AB-PAS-positive goblet cells in bronchioles.
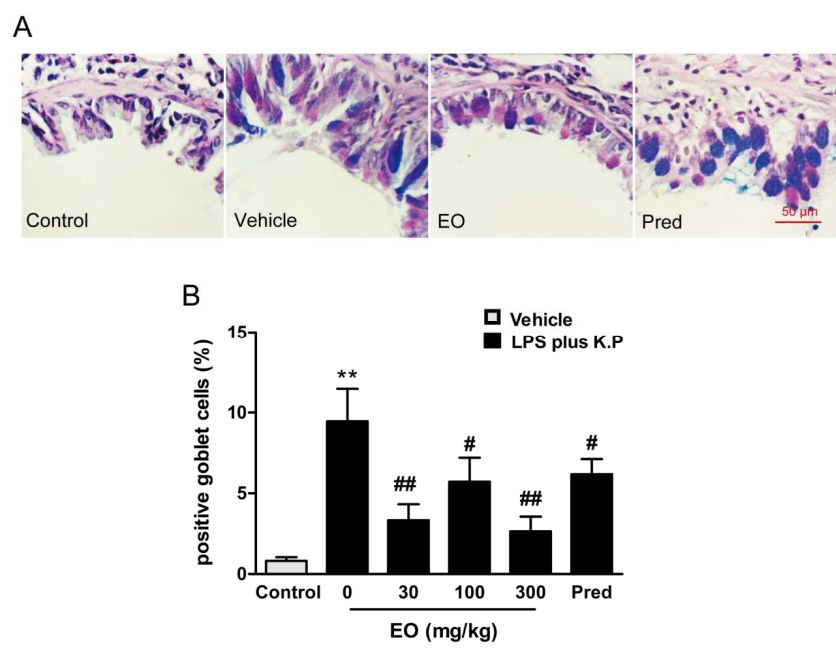

Figure 3. Effect of EO on changes of positive goblet cells in lungs. After challenged with vehicle or K. P plus LPS, lungs were immersed in $10 \%$ neutral formalin, paraffinized, and $5 \mu \mathrm{m}$ sections were prepared and stained with AB-PAS for examination of goblet cell hyperplasia (A). Positive goblet cell number and total cell number of columnar epithelial cells in the main bronchus and 2 3 medium bronchi were counted and analyzed (B). Data were expressed as mean $\pm S D$ of 6 rats /group. ${ }^{* *} P<0.01$ compared with control group, ${ }^{\#} P<0.01$ compared with vehicle group.

\subsection{Effect of Eucalyptus Oil on TNF- $\alpha$ and IL-1 $\beta$ Release}

The levels of TNF- $\alpha$ and IL-1 $\beta$ in lung homogenate were significantly increased after challenge of K. P plus LPS for 4 months. EO at dose of 30,100 and $300 \mathrm{mg} / \mathrm{kg}$ significantly reduced the production of pro-inflammatory cytokines TNF- $\alpha$ (Figure. 4A, P $<0.01$ ) and IL-1 $\beta$ (Figure. 4B, $\mathrm{P}<0.01$ ). Rats pretreated with Pred at dose of $10 \mathrm{mg} / \mathrm{kg}$ also showed decreases in TNF- $\alpha$ and IL- $1 \beta$ productions compared with vehicle group.
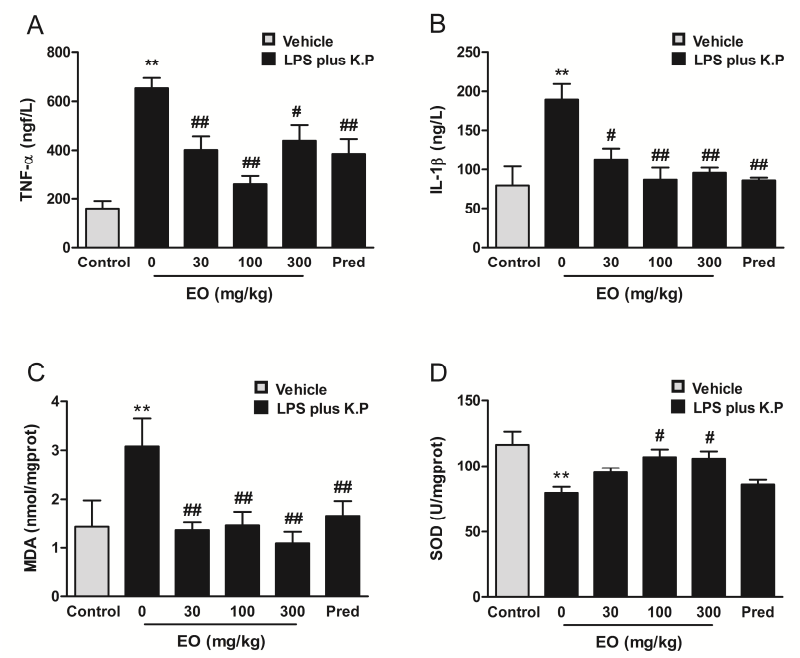

Figure 4. Effect of EO on TNF- $\alpha, I L-1 \beta, M D A$ production and SOD activity in lung homogenate. Lung tissues were harvested $24 \mathrm{~h}$ after the last $K . P$ exposure and used for determining the activity of TNF- $\alpha(A), I L-1 \beta(B), M D A$ $(C)$ and $S O D$ activity $(D)$ among groups. Data were expressed as mean $\pm S D$ of $8-12$ rats/group. ${ }^{* *} P<0.01$ compared with control group, ${ }^{\#} P<0.05,{ }^{\#} P<$ 0.01 compared with vehicle group.

\subsection{Effect of EO on MDA Production and SOD Activity in Lung Tissues}

After exposure with LPS plus K. P, rats produced a greater amount of MDA in vehicle group compared with control group. EO at 30,100 and $300 \mathrm{mg} / \mathrm{kg}$ significantly decreased the LPS plus K. P-induced MDA production in lung homogenate (Figure. $4 \mathrm{C}, \mathrm{P}<0.01$ ). In contrast, SOD activity was elevated obviously in vehicle group compared with that of control group. While EO at 100 and $300 \mathrm{mg} / \mathrm{kg}$ significantly increased the SOD activity in parallel to vehicle group (Figure. $4 \mathrm{D}, \mathrm{P}<0.05)$. These data indicated that EO might attenuate the LPS plus K. P-induced changes via oxidant-antioxidant balance. Among all the tested doses of EO, $100 \mathrm{mg} / \mathrm{kg}$ exhibited the best anti-oxidant effect. Pred at dose of $10 \mathrm{mg} / \mathrm{kg}$ decreased the MDA production but no significant effect on SOD activity.

\section{Discussion}

The present study aimed to evaluate the protective effect of EO using a LPS plus K. P-induced COPD model and to investigate its underlying mechanisms of the action associated with its anti-COPD activity.

Although cigarette smoking is the leading risk factor of COPD, only $15-20 \%$ of smokers develop the disease. It was postulated that bacterial infections play a major role in the pathogenesis of COPD [10]. Recently increasing evidence supported a clear relationship between bacteria infection and exacerbations of COPD, which is strongly associated with mucosal response and neutrophilic inflammatory profile in the sputum [11-13]. Here we established a COPD rat model using LPS plus K. P, characterized by chronic airway inflammation, emphysema and excess mucus. Oral administration of EO effectively reversed chronic bronchiolitis, with significant reduction of mucus hypersecretion. 
Treatment with EO for one month obviously attenuated the emphysematous changes and thickness of bronchioles while the vehicle group rats showed severe disruption of alveoli and thickened bronchioles. Our findings demonstrated that EO had protective effect against LPS plus K. P-induced pulmonary inflammation and destruction.

It's widely accepted that chronic airway inflammation plays a key role in the pathogenesis of COPD, associated with destruction of airway and lung tissues [14]. Previous evidence showed inflammatory cells such as neutrophils, macrophages and lymphocytes was aggregated in blood, sputum and BALF in COPD patients [15]. Release of inflammatory cytokines including TNF- $\alpha$, IL-1 $\beta$, IL-6 significantly increased in blood and lung tissues $[16,17]$. Here we detected the elevated extent of TNF- $\alpha$ and IL-1 $\beta$ in lung homogenate, which was obviously reduced by EO treatment in rats. In some trials, anti-inflammatory and antiseptic effect of EO and its extract has been identified [18-20]. Eucalyptol may regulate cytokine production in the airway through $\mathrm{TLR}_{4} / \mathrm{NF}-\kappa \mathrm{B}$ pathway, the mechanisms were to further indentify [21].

The lungs of patients with COPD are easy to breed micro-organisms including bacteria and virus infections. This results in further lung destruction and host denfenses decrease. Bacteria colonization and invasion constitute to a lazy immune response, which concomitantly occurs with oxidative stress (O'Rourke et al., 2003). The increased oxidants is very likely to tip the oxidant/antioxidant balance due to the existence of infection and increased immune response [22]. On the other hand, organisms have its own enzymatic and non-enzymatic defenses, such as Glutathione peroxidase (GSH-Px) and SOD against reactive oxidative stress and lipid peroxidation. To address the role of oxidative stress in our model, we addressed the release of MDA, an index of lipid peroxidation, and SOD activity in lung tissues of rats [23]. LPS plus K. P obviously increased MDA level, accompanied with decreased SOD activity, suggesting a critical role of oxidative stress in COPD model. However, treatment with EO significantly resulted in a significant decrease of MDA formation and increase of SOD activity. Our results indicate that EO potentially exerted protective effect against lung destruction via antioxidant mechanism.

Compositions of the major component of EO have been reported differently. Ben Hassine et al. found $\alpha$-pinene and 1,8-cineol as major components [24], and Bouzabata A et al. found mainly $\alpha$ - and $\beta$-pinene [25]. Whatever, EO extract is able to implement the innate cell-mediated immune response besides its anti-septic properties. In LPS-induced chronic bronchitis rats, EO reduced pulmonary inflammation and inhibited hypersecretion of airway mucins [26]. In accordance with this finding, our data supported the inhibition of mucus hypersecretion in COPD rats. Clinical trials have identified the benefit of concomitant therapy of cineol on improvement of lung function in patients with COPD [9]. Another placebo-controlled, double-blind trial also showed concomitant therapy using cineole improved lung function and health condition as well as to reduce dyspnea in asthma patients [27]. Our results provided direct evidence that EO attenuated lung destruction and chronic airway inflammation via anti-inflammatory and antioxidant properties, indicating EO as an active controller of lung injury in COPD.

Currently the clinical use of essential oils has expanded worldwide in the treatment of varieties of inflammatory diseases such as asthma and arthritis [28, 29]. EO has been traditionally used to treat respiratory tract disorders including bronchitis, pharyngitis and sinusitis. The scientific interest on medical plants in treating chronic pulmonary disease is expanding [30].

\section{Conclusion}

Our results provided direct evidence that available Eucalyptus oil might be a proper candidate drug in therapeutic of COPD, at least a good choice to concomitant therapy, apart from the clinical used for sputum clearance in treatment of airway disease.

\section{Acknowledgements}

This work was supported by the National Natural Science Foundation of China (No. 81341137, 31600386);. science and technology development program of traditional Chinese Medicine in Shandong province (2015-229).

\section{References}

[1] Samareh Fekri, M., et al., Detection of helicobacter pylori in bronchoalveolar lavage of patients with chronic obstructive pulmonary disease by real time polymerase chain reaction. Jundishapur J Microbiol, 2015. 8 (1): p. e14551.

[2] Cukic, V., The Most Common Detected Bacteria in Sputum of Patients with the Acute Exacerbation of COPD. Mater Sociomed, 2013. 25 (4): p. 226-9.

[3] Garcha, D. S., et al., Changes in prevalence and load of airway bacteria using quantitative PCR in stable and exacerbated COPD. Thorax, 2012. 67 (12): p. 1075-80.

[4] Korsgren, M., et al., Inhalation of LPS induces inflammatory airway responses mimicking characteristics of chronic obstructive pulmonary disease. Clin Physiol Funct Imaging, 2012. 32 (1): p. 71-9.

[5] Xu, H., M. Xiong, and Q. Huang, [The study on COPD rat model produced by bacterial infection]. Zhonghua Jie $\mathrm{He} \mathrm{He}$ Hu Xi Za Zhi, 1999. 22 (12): p. 739-42.

[6] Juergens, U. R., Anti-inflammatory properties of the monoterpene 1.8-cineole: current evidence for co-medication in inflammatory airway diseases. Drug Res (Stuttg), 2014. 64 (12): p. 638-46.

[7] Rantzsch, U., et al., Anti-inflammatory effects of Myrtol standardized and other essential oils on alveolar macrophages from patients with chronic obstructive pulmonary disease. Eur J Med Res, 2009. 14 Suppl 4: p. 205-9.

[8] Vigo, E., et al., In-vitro anti-inflammatory effect of Eucalyptus globulus and Thymus vulgaris: nitric oxide inhibition in J774A.1 murine macrophages. J Pharm Pharmacol, 2004. 56 (2): p. $257-63$. 
[9] Worth, H., C. Schacher, and U. Dethlefsen, Concomitant therapy with Cineole (Eucalyptole) reduces exacerbations in COPD: a placebo-controlled double-blind trial. Respir Res, 2009. 10: p. 69.

[10] Anthonisen, N. R., The British hypothesis revisited. Eur Respir J, 2004. 23 (5): p. 657-8.

[11] Sethi, S., et al., Strain-specific immune response to Haemophilus influenzae in chronic obstructive pulmonary disease. Am J Respir Crit Care Med, 2004. 169 (4): p. 448-53.

[12] Sethi, S., et al., Inflammatory profile of new bacterial strain exacerbations of chronic obstructive pulmonary disease. Am J Respir Crit Care Med, 2008. 177 (5): p. 491-7.

[13] Sethi, S., et al., New strains of bacteria and exacerbations of chronic obstructive pulmonary disease. N Engl J Med, 2002. 347(7): p. 465-71.

[14] Zanini, A., et al., Bronchial hyperresponsiveness, airway inflammation, and reversibility in patients with chronic obstructive pulmonary disease. Int J Chron Obstruct Pulmon Dis, 2015. 10: p. 1155-61.

[15] Mroz, R. M., et al., Anti-inflammatory effects of atorvastatin treatment in chronic obstructive pulmonary disease. A controlled pilot study. J Physiol Pharmacol, 2015. 66 (1): p. 111-28.

[16] Tang, Y., et al., The role of the serum IL-33/sST2 axis and inflammatory cytokines in chronic obstructive pulmonary disease. J Interferon Cytokine Res, 2014. 34 (3): p. 162-8.

[17] Shen, L. L., et al., Inhalation of glycopyrronium inhibits cigarette smoke-induced acute lung inflammation in a murine model of COPD. Int Immunopharmacol, 2014. 18 (2): p. 358-64.

[18] Tsai, M. L., et al., Antimicrobial, antioxidant, and anti-inflammatory activities of essential oils from five selected herbs. Biosci Biotechnol Biochem, 2011. 75 (10): p. 1977-83.

[19] Mulyaningsih, S., et al., Antibacterial activity of essential oils from Eucalyptus and of selected components against multidrug-resistant bacterial pathogens. Pharm Biol, 2011. 49 (9): p. 893-9.
[20] Cermelli, C., et al., Effect of eucalyptus essential oil on respiratory bacteria and viruses. Curr Microbiol, 2008. 56 (1): p. 89-92.

[21] Zhao, C., et al., 1,8-cineol attenuates LPS-induced acute pulmonary inflammation in mice. Inflammation, 2014. 37 (2): p. 566-72.

[22] van der Strate, B. W., et al., Cigarette smoke-induced emphysema: A role for the B cell? Am J Respir Crit Care Med, 2006. 173 (7): p. 751-8.

[23] Ismail, M., et al., Effect of spirulina intervention on oxidative stress, antioxidant status, and lipid profile in chronic obstructive pulmonary disease patients. Biomed Res Int, 2015. 2015: p. 486120.

[24] Ben Hassine, D., et al., Chemical composition and in vitro evaluation of the antioxidant and antimicrobial activities of Eucalyptus gillii essential oil and extracts. Molecules, 2012. 17 (8): p. 9540-58

[25] Bouzabata, A., et al., Composition and chemical variability of Eucalyptus bosistoana essential oil from Algerian Sahara. Nat Prod Commun, 2014. 9 (5): p. 701-2.

[26] Lu, X. Q., et al., [Effect of Eucalyptus globulus oil on lipopolysaccharide-induced chronic bronchitis and mucin hypersecretion in rats]. Zhongguo Zhong Yao Za Zhi, 2004. 29 (2): p. 168-71.

[27] Worth, H. and U. Dethlefsen, Patients with asthma benefit from concomitant therapy with cineole: a placebo-controlled, double-blind trial. J Asthma, 2012. 49 (8): p. 849-53.

[28] Shirole, R. L., et al., Investigation into the mechanism of action of essential oil of Pistacia integerrima for its antiasthmatic activity. J Ethnopharmacol, 2014. 153 (3): p. 541-51.

[29] Wang, C., et al., Treatment with total alkaloids from Radix Linderae reduces inflammation and joint destruction in type II collagen-induced model for rheumatoid arthritis. J Ethnopharmacol, 2007. 111 (2): p. 322-8.

[30] Ram, A., et al., Medicinal plants useful for treating chronic obstructive pulmonary disease (COPD): current status and future perspectives. Fitoterapia, 2011. 82 (2): p. 141-51. 